\title{
PERTURBATION THEORY FOR SEMI-GROUPS OF LINEAR OPERATORS
}

\author{
BY
}

\section{R. S. PHILLIPS}

1. Introduction. Perturbation theory has long been a very useful tool in the hands of both the analyst and the physicist. A considerable amount of research has been done on the perturbation of self-adjoint linear operators on a Hilbert space, principally by F. Rellich [4](1). J. D. Newburgh [3] and F. Wolf [7] have investigated a perturbation theory for general closed linear operators on a Banach space to itself and it appears that much of the selfadjoint theory does not carry over to the general situation. In this paper we are concerned with a different aspect of perturbation theory, namely, the effect on a semi-group of linear bounded operators (see Hille [2]) of adding a bounded linear operator to the infinitesimal generator of the semi-group. The insistence that the perturbing operator be bounded is an unpleasant restriction. However, the simplicity and wealth of detail of the results for the linear bounded perturbation to some extent atone for this lack of generality; and since the methods do not seem to be adequate for a more general type of perturbation, we feel justified in limiting this paper to the bounded case.

Those properties of a semi-group which persist under a linear bounded perturbation of the infinitesimal generator we shall call stable properties. In practical applications one would expect that the infinitesimal generator itself is known only to within certain limits of error and hence that a physical significance could be attached only to the stable properties of the associated semi-group of transformations. Mathematically one would expect that the stable properties of a semi-group are more basic than the others and that the significant theorems in the subject should evolve about these properties. In this paper we have made a start at cataloguing the stable and unstable semi-group properties.

We first obtain a necessary and sufficient condition that a closed linear operator on a Banach space $\mathfrak{X}$ to itself generate a semi-group (or group) of bounded linear transformations strongly convergent to the identity at the origin. By means of this result we are able to show in section three that if $A$ generates a semi-group (or group) of this type, then so does $A+B$ where $B$ is any bounded linear transformation on $\mathfrak{X}$ to itself. Thus the property of being a semi-group or group of transformations is itself stable. If $A$ generates $T(s)$ and $A+B$ generates $S(s)$, then the latter semi-group can be represented

Presented to the Society, May 3, 1952; received by the editors April 4, 1952.

(1) Numbers in brackets refer to the references cited at the end of the paper. 
by an absolutely and uniformly (in every finite interval) convergent series $S(s)=\sum_{n=0}^{\infty} S_{n}(s)$ where $S_{0}(s)=T(s)$ and $S_{n}(s)=\int_{0}^{s} T(s-\sigma) B S_{n-1}(\sigma) d \sigma$. If $\|T(s)\|$ satisfies an inequality of the type $\|T(s)\| \leqq \exp (\omega s)$, then so does $\|S(s)\|$. Moreover if $T(s)$ is uniformly continuous for $s>0$, then so is $S(s)$. However the property of being eventually uniformly continuous is not stable (see §5). If $T(z)$ is holomorphic in a sector $\Phi_{1}<\phi<\Phi_{2}\left(-\pi / 2 \leqq \Phi_{1}<0<\Phi_{2}\right.$ $\leqq \pi / 2)$ and bounded near the origin in every subsector $\Phi_{1}+\epsilon<\phi<\Phi_{2}-\epsilon$ $(\epsilon>0)$, then the same is true of $S(z)$. In $\$ 4$ we consider the resolvent of $A$ to be either completely continuous or weakly completely continuous. These are likewise stable semi-group properties. Further if $A$ is any closed linear operator with completely continuous resolvent, then $A$ has a pure point spectrum consisting only of isolated points and it is possible to develop a simple "finite" elementary divisor theory for $A$.

$\$ 5$ is devoted to showing that certain semi-group properties are not stable. The property that the elements of the semi-group are generalized nilpotent is not stable nor is the property that the eigen-spaces $\left({ }^{2}\right)$ span the space.

The semi-group of operators $T(s)(s \in[0, \infty))$ is a strongly continuous one-parameter family of bounded linear operators on $\mathfrak{X}$ to itself which solves the differential equation $d T(s) x / d s=A T(s) x$ for $x$ in the domain $\mathfrak{D}(A)$ of $A$ with the initial condition $T(0)=I$. In $\S 6$ we consider a natural generalization of this, namely, the case where $A$ varies with $s$. We have supposed that $A(s)$ $=A+B(s)$ where $A$ is the infinitesimal generator of a semi-group of operators and $B(s)$ is a strongly continuously differentiable one-parameter family of bounded linear operators. In this case there exists a unique strongly continuous one-parameter family of bounded linear operators $U(s)$ such that $d U(s) x / d s=A(s) U(s) x$ for all $x \in \mathfrak{D}(A)$ and such that $U(0)=I$. We also treat the nonhomogeneous differential equation.

2. On the generation of groups and semi-groups of operators. Let $\mathfrak{X}$ be a complex Banach space and let $\mathbb{E}(\mathfrak{X})$ be the Banach algebra of all bounded linear transformations on $\mathfrak{X}$ to itself. We shall be concerned with a semi-group of operators (see [2]) on a semi-module $\subseteq$ to $\mathfrak{E}(\mathfrak{X})$ satisfying the following hypothesis.

Hypothesis $\mathrm{H}$.

$$
\begin{aligned}
T\left(s_{1}+s_{2}\right) & =T\left(s_{1}\right) T\left(s_{2}\right), \quad s_{1}, s_{2} \in \mathfrak{S}, \\
T(0) & =I .
\end{aligned}
$$

(ii) $T(s)$ is strongly continuous on $\mathfrak{S}$.

At various times $\subseteq$ will be the half-line $[0, \infty)$; or the whole line $(-\infty, \infty)$; or a sector in the complex plane $\Phi_{1}<\arg s<\Phi_{2},-\pi / 2 \leqq \Phi_{1}<0<\Phi_{2} \leqq \pi / 2$; or some $k$.

(2) The eigenspace associated with $\lambda_{0}$ is the set of all vectors $x$ such that $\left(\lambda_{0} I-A\right)^{k} x=0$ for 
the whole complex plane. For the present we suppose $S$ to be $[0, \infty)$. Then it is readily seen that $\omega(s)=\log \|T(s)\|$ is a lower semi-continuous subadditive function and hence that

$$
\omega_{0}=\inf _{s>0} \omega(s) / s=\lim _{s \rightarrow \infty} \omega(s) / s .
$$

Further given $\epsilon>0$, there exists an $M_{\epsilon}>0$ such that

$$
\|T(s)\| \leqq M_{\epsilon} \exp \left[\left(\omega_{0}+\epsilon\right) s\right] .
$$

Let $A$ be the infinitesimal generator of the semi-group $T(s)$. Then $A$ will be a closed linear operator, in general unbounded, with domain $\mathfrak{D}(A)$ dense in $\mathfrak{X}$. The resolvent $R(\lambda ; A)$ of $A$ belongs to $\mathscr{E}(\mathfrak{X})$ and is defined and holomorphic on the resolvent set for $A$, namely $\rho(A)$. For $\lambda$ and $\zeta$ in this set, the resolvent satisfies the first resolvent equation

$$
R(\lambda ; A)-R(\zeta ; A)=(\zeta-\lambda) R(\lambda ; A) R(\zeta ; A)
$$

as well as the defining relations

$$
\begin{aligned}
(\lambda I-A) R(\lambda ; A) & =I \\
R(\lambda ; A)(\lambda I-A) x & =x
\end{aligned}
$$$$
\text { for } x \in \mathfrak{D}(A) \text {. }
$$

For $\operatorname{Re}(\lambda)>\omega_{0}$, we have

$$
R(\lambda ; A) x=\int_{0}^{\infty} \exp (-\lambda s) T(s) x d s .
$$

The spectrum of $A$, denoted by $\Sigma(A)$, is by definition the complementary set to $\rho(A)$.

Our first theorem is a generalization of a result due to both E. Hille [2, Theorem 12.2.1] and to K. Yosida [8] on the generation of semi-groups.

Theorem 2.1. A necessary and sufficient condition that a closed linear operator $A$ with dense domain generate a semi-group satisfying hypothesis $(\mathrm{H})$ on $[0, \infty)$ is that there exist real numbers $M>0$ and $\omega$ such that

$$
\left\|R(\lambda ; A)^{n}\right\| \leqq M(\lambda-\omega)^{-n} \quad \text { for } \lambda>\omega .
$$

The necessity readily follows from the relations (2) and (5). In fact, making use of the operational calculus $\left[2\right.$, p. 304], we see that for $\lambda>\omega>\omega_{0}$ we have

$$
R(\lambda ; A)^{n} x=[(n-1) !]^{-1} \int_{0}^{\infty} \exp (-\lambda s) s^{n-1} T(s) x d s
$$

and hence that

$$
\left\|R(\lambda ; A)^{n}\right\| \leqq[(n-1) !]^{-1} \int_{0}^{\infty} \exp (-\lambda s) s^{n-1}\|T(s)\| d s \leqq M(\lambda-\omega)^{-n}
$$


Both the method of Hille [2] and that of Yosida [8] will yield the sufficiency argument. For the sake of completeness we include a proof modelled after the Yosida proof. Let $B_{\lambda}=-\lambda[I-\lambda R(\lambda ; A)]$. Then

$$
\exp \left(s B_{\lambda}\right)=\exp (-\lambda s) \sum_{n=0}^{\infty}\left(\lambda^{2} s\right)^{n} R(\lambda ; A)^{n} / n !
$$

so that

$$
\begin{aligned}
\left\|\exp \left(s B_{\lambda}\right)\right\| & \leqq \exp (-\lambda s) \sum_{n=0}^{\infty}\left(\lambda^{2} s\right)^{n}\left[M(\lambda-\omega)^{-n}\right] / n ! \\
& \leqq M \exp \left[s \omega \lambda(\lambda-\omega)^{-1}\right] \leqq M \exp \left(s \omega^{\prime}\right)
\end{aligned}
$$

for $\lambda$ sufficiently large (depending on our choice of $\omega^{\prime}>\omega_{0}$ ). We show next that $\lim _{\lambda \rightarrow \infty} B_{\lambda} x=A x$ for $x \in \mathfrak{D}(A)$. By (4),

$$
\|\lambda R(\lambda ; A) x-x\|=\|R(\lambda ; A) A x\| \leqq M\|A x\|(\lambda-\omega)^{-1} \rightarrow 0 \quad \text { as } \lambda \rightarrow \infty .
$$

Since $\|\lambda R(\lambda ; A)\| \leqq K$ for $\lambda>2 \omega$ and since $\mathfrak{D}(A)$ is dense in $\mathfrak{X}$, it follows by the Banach-Steinhaus theorem [1, p. 79] that $\lim _{\lambda \rightarrow \infty} \lambda R(\lambda ; A) x=x$ for all $x \in \mathfrak{X}$. Thus in particular for $x \in \mathfrak{D}(A), B_{\lambda} x=\lambda R(\lambda ; A) A x \rightarrow A x$ as $\lambda \rightarrow \infty$. We next set $S_{\lambda}(s)=\exp \left(s B_{\lambda}\right)$. Then for $x \in \mathfrak{D}(A)$ we have

$$
\begin{aligned}
S_{\lambda}(s) x-S_{\mu}(s) x & =\int_{0}^{s} \frac{d}{d \sigma}\left[S_{\mu}(s-\sigma) S_{\lambda}(\sigma) x\right] d \sigma \\
& =\int_{0}^{s} S_{\mu}(s-\sigma) S_{\lambda}(\sigma)\left(B_{\lambda} x-B_{\mu} x\right) d \sigma .
\end{aligned}
$$

Making use of (7) we obtain

$$
\left\|S_{\lambda}(s) x-S_{\mu}(s) x\right\| \leqq M^{2} s \exp \left(s \omega^{\prime}\right)\left\|B_{\lambda} x-B_{\mu} x\right\| .
$$

Thus $S_{\lambda}(s) x$ converges uniformly in every finite interval to a limit which we designate by $T(s) x$. Since $\mathfrak{D}(A)$ is dense in $\mathfrak{X}$, it follows again by the BanachSteinhaus theorem (and by (7)) that $S_{\lambda}(s) x$ converges uniformly in every finite interval to a limit for all $x \in \mathfrak{X}$. Further since $S_{\mathrm{\lambda}}(s)$ is a semi-group of bounded operators strongly continuous on $[0, \infty)$, the same is true of $T(s)$. Finally, taking the limit on both sides of $S_{\lambda}(s) x-x=\int_{0}^{s} S_{\lambda}(\sigma) B_{\lambda} x d \sigma$, we see that for $x \in \mathfrak{D}(A)$

$$
T(s) x-x=\int_{0}^{s} T(\sigma) A x d \sigma .
$$

Thus if $B$ is the infinitesimal generator of $T(s)$, the differentiating of (8) gives us $B x=A x$ on $\mathfrak{D}(A) \subset \mathfrak{D}(B)$. Now for $\lambda>\omega, R(\lambda ; B)$ is an inverse and $R(\lambda ; A)$ is a right inverse for $(\lambda I-B)$. Hence $R(\lambda ; B)=R(\lambda ; A)$ and $A \equiv B$. 
Corollary 2.2. A necessary and sufficient condition that a closed linear operator $A$ with dense domain generate a semi-group $T(s)$ satisfying $(\mathrm{H})$ on $[0, \infty)$ and such that $\|T(s)\| \leqq \exp (\omega s)$ is that

$$
\|R(\lambda ; A)\| \leqq(\lambda-\omega)^{-1}
$$

for $\lambda>\omega$.

The necessity follows directly from (5). It is clear that (9) implies (6) with $M=1$. Again since $M=1$ in (7), the same proof as in Theorem 2.1 will yield $T(s)$ with $\|T(s)\| \leqq \exp \left(\omega^{\prime} s\right)$ for all $\omega^{\prime}>\omega$ and hence for $\omega^{\prime}=\omega$.

Corollary 2.2 is a slightly sharpened statement of the Hille-Yosida theorem. It is worth noting that the condition $\|T(s)\| \leqq \exp (\omega s)$ is a stronger condition than $\lim \|T(s)\|=1$. For example if we define $\alpha(s)=\left(2 s-s^{2}\right)^{1 / 2}$ for $0 \leqq s \leqq 2$ and equal to zero elsewhere, then it is easy to see that $\alpha(s)$ is subadditive and continuous on $[0, \infty)$. Hence by a theorem due to Hille [2, Theorem 16.3.1] there exists a semi-group of transformations satisfying $(\mathrm{H})$ on $[0, \infty)$ such that $\|T(s)\|=\exp [\alpha(s)]$. It is clear in this case that $\|T(s)\|$ is not dominated by $\exp (\omega s)$ for any $\omega$.

TheOREM 2.3. A necessary and sufficient condition that a closed linear operator $A$ with dense domain generate a group satisfying $(\mathrm{H})$ on $(-\infty, \infty)$ is that there exist real numbers $M>0$ and $\omega \geqq 0$ such that

$$
\left\|R(\lambda ; A)^{n}\right\| \leqq M(|\lambda|-\omega)^{-n} \quad \text { for } \lambda>\omega \text { and } \lambda<-\omega .
$$

The necessity follows as in the proof on Theorem 2.1. Further since $R(\lambda ;-A)=-R(-\lambda ; A)$, it follows from Theorem 2.1 that both $A$ and $-A$ generate semi-groups, say $T_{+}(s)$ and $T_{-}(s)$ respectively. It is clear from the proof that the approximating semi-groups commute and hence that $T_{+}(s)$ and $T_{-}(s)$ likewise commute. Thus $S(s)=T_{+}(s) T_{-}(s)$ is also a semi-group satisfying $(\mathrm{H})$ on $[0, \infty)$. For $x \in \mathfrak{D}(A)$, it is easy to see that $d S(s) x / d s=0$. Thus $S(s) x=x$ for $x \in \mathfrak{D}(A)$ and all $s \geqq 0$, and since $\mathfrak{D}(A)$ is dense in $\mathfrak{X}$ the same is true for all $x \in \mathfrak{X}$, that is, $T_{-}(s)=T_{+}(s)^{-1}$. Therefore $T(s)=T_{+}(s)$ for $s \geqq 0$ and $T_{-}(s)$ for $s \leqq 0$ defines a group of linear operators satisfying $(\mathrm{H})$ on $(-\infty, \infty)$ with infinitesimal generator $A$.

3. Stable properties. We now make use of the foregoing results to develop a perturbation theory for semi-groups of operators. We intend to perturb the infinitesimal generator by adding to it a linear bounded operator. This section will deal with those properties of a semi-group which persist under such a perturbation.

Lemma 3.1. Let $A$ be a closed linear operator on $\mathfrak{X}$ to $\mathfrak{X}$ and suppose $B \in \mathbb{E}(\mathfrak{X})$ is such that $\left\|B R\left(\lambda_{0} ; A\right)\right\|=\gamma<1$ for some $\lambda_{0} \in \rho(A)$. Then $A+B$ is a closed linear operator with domain $\mathfrak{D}(A)$ and $R\left(\lambda_{0} ; A+B\right)$ exists and

$$
R\left(\lambda_{0} ; A+B\right)=\sum_{n=0}^{\infty} R\left(\lambda_{0} ; A\right)\left[B R\left(\lambda_{0} ; A\right)\right]^{n}
$$


Further $\left\|R\left(\lambda_{0} ; A\right)-R\left(\lambda_{0} ; A+B\right)\right\| \leqq\left\|R\left(\lambda_{0} ; A\right)\right\| \gamma(1-\gamma)^{-1}$.

It is clear that $A+B$ is a closed linear operator with domain $\mathfrak{D}(A)$. We note that

$$
R \equiv \sum_{n=0}^{\infty} R\left(\lambda_{0} ; A\right)\left[B R\left(\lambda_{0} ; A\right)\right]^{n}=R\left(\lambda_{0} ; A\right)\left[I-B R\left(\lambda_{0} ; A\right)\right]^{-1}
$$

and hence that

$$
\left[\lambda_{0} I-(A+B)\right] R=\left[I-B R\left(\lambda_{0} ; A\right)\right]\left[I-B R\left(\lambda_{0} ; A\right)\right]^{-1}=I .
$$

Further the range of $R$ is precisely $\mathfrak{D}(A)$ since the range of $\left[I-B R\left(\lambda_{0} ; A\right)\right]^{-1}$ is $\mathfrak{X}$. Thus given $x \in \mathfrak{D}(A)$ there exists a $y$ such that $x=R y$. Therefore

$$
R\left[\lambda_{0} I-(A+B)\right] x=R\left[\lambda_{0} I-(A+B)\right] R y=R y=x
$$

so that $R$ is both a left and a right inverse. The bound on $\| R\left(\lambda_{0} ; A\right)$ $-R\left(\lambda_{0} ; A+B\right) \|$ comes directly from the expansion.

THEOREM 3.2. If $A$ is the infinitesimal generator of a semi-group of linear operators satisfying $(H)$ on $[0, \infty)$ and if $B \in \mathfrak{E}(\mathfrak{X})$, then $A+B$ on $\mathfrak{D}(A)$ is likewise the infinitesimal generator of a semi-group of linear operators satisfying $(H)$ on $[0, \infty)$.

By Theorem 2.1, $R(\lambda ; A)$ satisfies the inequalities (6). Hence for $\lambda>\omega_{1}$ $=\omega+M\|B\|,\|B R(\lambda ; A)\| \leqq M\|B\| /(\lambda-\omega)<1$; and by Lemma 3.1, $R(\lambda ; A+B)$ exists and is equal to $\sum_{j=0}^{\infty} R(\lambda ; A)[B R(\lambda ; A)]^{i}$ which converges absolutely. We now regroup the terms of $\left[\sum_{j=0}^{\infty} R(\lambda ; A)[B R(\lambda ; A)]^{i}\right]^{n}$ according to powers of $B$. Any term containing $k$ of the $B$ 's will possess $n+k R(\lambda ; A)$ 's since each $B$ introduces another $R(\lambda ; A)$. Further the $R(\lambda ; A)$ 's will be in $k+1$ nonempty groups separated from each other by the $k B$ 's. In other words it will be of the form

$$
R(\lambda ; A)^{r_{1}} B R(\lambda ; A)^{r_{2}} B \cdots R(\lambda ; A)^{r_{k}} B R(\lambda ; A)^{r_{k+1}},
$$

$\sum_{i=1}^{k+1} r_{i}=n+k$ and $r_{i}>0$. In norm this term will be less than or equal to

$$
\begin{array}{r}
M(\lambda-\omega)^{-r_{1}}\|B\| M(\lambda-\omega)^{-r_{2}}\|B\| \cdots M(\lambda-\omega)^{-r_{k}}\|B\| M(\lambda-\omega)^{-r k+1} \\
=M^{k+1}\|B\| \|^{k}(\lambda-\omega)^{-(n+k)} .
\end{array}
$$

The number of terms containing $k$ of the $B$ 's is precisely the coefficient of $x^{k}$ in $(1-x)^{-n}=\sum_{k=0}^{\infty} C_{k}^{n} x^{k}$. Hence

$$
\begin{aligned}
\left\|R(\lambda ; A+B)^{n}\right\| & \leqq \sum_{k=0}^{\infty} C_{k}^{n} M^{k+1}\|B\|^{k}(\lambda-\omega)^{-(n+k)} \\
& =M(\lambda-\omega)^{-n}\left[1-M\|B\|(\lambda-\omega)^{-1}\right]^{-1} \leqq M\left(\lambda-\omega_{1}\right)^{-n}
\end{aligned}
$$

for $\lambda>\omega_{1}$. The result now follows from Theorem 2.1. 
THEOREM 3.3. Let $A$ be the infinitesimal generator of a semi-group of linear operators $T(s)$ satisfying $(\mathrm{H})$ on $[0, \infty)$ and such that $\|T(s)\| \leqq \exp (\omega s)$. If $B \in \mathbb{E}(\mathfrak{X})$, then $A+B$ on $\mathfrak{D}(A)$ is likewise the infinitesimal generator of a semigroup of linear operators $S(s)$ satisfying $(\mathrm{H})$ on $[0, \infty)$ and such that $\|S(s)\|$ $\leqq \exp \left(\omega_{1} s\right)$ where $\omega_{1}=\omega+\|B\|$.

In the proof of Theorem 3.2 we have merely to replace $M$ by 1 to obtain the inequality $\|R(\lambda, A+B)\| \leqq\left(\lambda-\omega_{1}\right)^{-1}$ for $\lambda>\omega_{1}$. The result now follows from Corollary 2.2.

THEOREM 3.4. If $A$ is the infinitesimal generator of a group of linear operators satisfying $(\mathrm{H})$ on $(-\infty, \infty)$ and if $B \in \mathbb{E}(\mathfrak{X})$, then $A+B$ on $\mathfrak{D}(A)$ is likewise the infinitesimal generator of a group of linear operators satisfying (H) on $(-\infty, \infty)$.

The proof follows the same argument as that of Theorem 3.2, depending now on Theorem 2.3.

TheOREM 3.5. If $A$ is the infinitesimal generator of a semi-group of linear operators $T(s)$ satisfying $(\mathrm{H})$ on $[0, \infty)$ and if $B \in \mathbb{E}(\mathfrak{X})$, then the semi-group of linear operators $S(s)$ generated by $A+B$ (defined on $\mathfrak{D}(A)$ ) can be represented by the series expansion

$$
S(s)=\sum_{n=0}^{\infty} S_{n}(s)
$$

where $S_{0}(s)=T(s)$ and $S_{n}(s) x=\int_{0}^{s} T(s-\sigma) B S_{n-1}(\sigma) x d \sigma$.

It is clear that $S_{0}(s)$ is strongly continuous on $[0, \infty)$ and that $\left\|S_{0}(s)\right\|$ $\leqq M \exp (\omega s)$ by (2). Suppose $S_{n}(s)$ is likewise strongly continuous on $[0, \infty)$ and that

$$
\left\|S_{n}(s)\right\| \leqq M(M\|B\|)^{n} s^{n} \exp (\omega s) / n ! .
$$

Then $T(s-\sigma) B S_{n}(\sigma)$ will be strongly continuous on $[0, s]$ so that the integral defining $S_{n+1}(s)$ exists in the strong topology. Further

$$
\begin{aligned}
\left\|S_{n+1}(s)\right\| & \leqq M\|B\| \int_{0}^{s} \exp [\omega(s-\sigma)]\left\|S_{n}(\sigma)\right\| d \sigma \\
& \leqq M(M\|B\|)^{n+1} s^{n+1} \exp (\omega s) /(n+1) !
\end{aligned}
$$

Finally for $s_{1}<s_{2}$

$$
\begin{aligned}
\left\|S_{n+1}\left(s_{2}\right) x-S_{n+1}\left(s_{1}\right) x\right\| \leqq & \int_{0}^{s_{1}}\left\|\left[T\left(s_{2}-\sigma\right)-T\left(s_{1}-\sigma\right)\right] B S_{n}(\sigma) x\right\| d \sigma \\
& +\int_{0}^{s 2}\left\|T\left(s_{2}-\sigma\right)\right\|\|B\|\left\|S_{n}(\sigma) x\right\| d \sigma
\end{aligned}
$$


As $s_{1}, s_{2} \rightarrow s_{0}$, the integrand in the first term on the right converges to zero boundedly and the integrand of the second term is bounded. It follows that $S_{n+1}(s)$ is strongly continuous on $[0, \infty)$. By induction, $S_{n}(s)$ is well defined, strongly continuous, and satisfies (13) for all integers $n$. Thus the series (12) is majorized by the series expansion of $M \exp \left(\omega_{1} s\right)$ where $\omega_{1}=\omega+M\|B\|$. For $\lambda>\omega_{1}$, we can therefore write

$$
\int_{0}^{\infty} \exp (-\lambda s)\left[\sum_{n=0}^{\infty} S_{n}(s) x\right] d s=\sum_{n=0}^{\infty} \int_{0}^{\infty} \exp (-\lambda s) S_{n}(s) x d s .
$$

Now if $x^{*} \in X^{*}$ it is a consequence of the strong convergence of the integral and of the Fubini theorem that

$$
\begin{aligned}
x^{*}\left[\int_{0}^{\infty} \exp \right. & \left.(-\lambda s) S_{n}(s) x d s\right] \\
& =\int_{0}^{\infty} \exp (-\lambda s) x^{*}\left[S_{n}(s) x\right] d s \\
& =\int_{0}^{\infty} \exp (-\lambda s) \int_{0}^{\infty} x^{*}\left[T(s-\sigma) B S_{n-1}(\sigma) x\right] d \sigma d s \\
& =\int_{0}^{\infty} \exp (-\lambda \sigma) \int_{0}^{\infty} \exp [-\lambda(s-\sigma)] x^{*}\left[T(s-\sigma) B S_{n-1}(\sigma) x\right] d s d \sigma \\
& =\int_{0}^{\infty} \exp (-\lambda \sigma) x^{*}\left[R(\lambda ; A) B S_{n-1}(\sigma) x\right] d \sigma \\
& =x^{*}\left[R(\lambda ; A) B\left\{\int_{0}^{\infty} \exp (-\lambda \sigma) S_{n-1}(\sigma) x d \sigma\right\}\right] .
\end{aligned}
$$

Hence by induction

$$
\int_{0}^{\infty} \exp (-\lambda s) S_{n}(s) x d s=R(\lambda ; A)[B R(\lambda ; A)]^{n} x .
$$

From the proof of Theorem 3.2 it is known that

$$
R(\lambda ; A+B) x=\int_{0}^{\infty} \exp (-\lambda s) S(s) x d s=\sum_{n=0}^{\infty} R(\lambda ; A)[B R(\lambda ; A)]^{n} x .
$$

Thus for $\lambda>\omega_{1}$, the Laplace transforms of both $S(s)$ and $\sum_{n=0}^{\infty} S_{n}(s)$ are equal and hence by the uniqueness theorem [2, Theorem 10.2.3] these two functions are equal.

The formal expansion (12) is familiar to the physicist for groups of unitary operators on a Hilbert space. By means of the inequalities (13) we are also able to estimate the remainder term if we use only a finite number of 
terms in the expansion (12).

CoROLlaRy 3.6. Under the hypothesis of the preceding theorem

$$
\left|S(s)-\sum_{k=0}^{n} S_{k}(s)\right| \leqq M(M\|B\|)^{n+1} s^{n+1} \exp (\omega, s) /(n+1) !
$$

where $M$ depends on the choice of $\omega>\omega_{0}$ and $\omega_{1}=\omega+M\|B\|$. In particular $\|S(s)-T(s)\| \leqq M^{2}\|B\| s \exp (\omega, s)$.

COROLLARY 3.7. If $A$ is the infinitesimal generator of a semi-group of linear operators $T(s)$ satisfying $(H)$ on $[0, \infty)$ and if $B(z) \in \mathbb{E}(\mathfrak{X})$ is holomorphic in some domain \&s of the complex plane, then the semi-group of linear operators $S(z, s)$ generated by $A+B(z)$ is likewise holomorphic in (S) for each $s \geqq 0$.

It follows from (13) that the expansion $\sum_{n=0}^{\infty} S_{n}(z, s)$ converges uniformly in every closed bounded subdomain of (S). It is therefore sufficient to show that each $S_{n}(z, s)$ is holomorphic in (\$). This is clearly true of $S_{0}(z, s)=T(s)$. Suppose it is likewise true for $S_{n}(z, s)$. Then for $z_{1}, z_{2} \in(S$,

$$
\begin{aligned}
\frac{S_{n+1}\left(z_{2}, s\right)-S_{n+1}\left(z_{1}, s\right)}{z_{2}-z_{1}}= & \int_{0}^{s} T(s-\sigma) \frac{B\left(z_{2}\right)-B\left(z_{1}\right)}{z_{2}-z_{1}} S_{n}\left(z_{2}, \sigma\right) d \sigma \\
& +\int_{0}^{8} T(s-\sigma) B\left(z_{1}\right) \frac{S_{n}\left(z_{2}, \sigma\right)-S_{n}\left(z_{1}, \sigma\right)}{z_{2}-z_{1}} d \sigma .
\end{aligned}
$$

This converges to a limit in the uniform topology as $z_{1} \rightarrow z_{2}$ since this is true of the incremental ratios on the right and since $\left[S_{n}\left(z_{2}, s\right)-S_{n}\left(z_{1}, s\right)\right] /\left(z_{2}-z_{1}\right)$ converges uniformly in $s \in[0, L]$ to its limit (because of the fact that $S_{n}(z, s)$ with $s \in[0, L]$ is uniformly bounded in every closed bounded subdomain of (अ).

THEOREM 3.8. Let $A$ be the infinitesimal generator of a semi-group of linear transformations $T(s)$ satisfying $(\mathrm{H})$ on $[0, \infty)$ and uniformly continuous for $s>0$. Then for $B \in \mathcal{E}(\mathfrak{X})$, the semi-group of linear operators $S(s)$ generated by $A+B$ on $\mathfrak{D}(A)$ is likewise uniformly continuous for $s>0$.

Here again we make use of the series representation (12) for $S(s)$. Because of (13) the expansion converges in the uniform operator topology uniformly in every finite interval. Thus it is sufficient to show that $S_{n}(s)$ is uniformly continuous for $s>0$. This is true by hypothesis for $n=0$. Again using an inductive argument we assume it to be true for $S_{n}(s)$. Then for $s_{1} \leqq s_{0} \leqq s_{2}$

$$
\begin{aligned}
& \left\|S_{n+1}\left(s_{2}\right)-S_{n+1}\left(s_{1}\right)\right\| \\
& \leqq \\
& \quad(M\|B\|)^{n+1}(n !)^{-1} \int_{0}^{s_{1}}\left\|T\left(s_{2}-\sigma\right)-T\left(s_{1}-\sigma\right)\right\| \sigma^{n} \exp (\omega \sigma) d \sigma \\
& \quad+\left|s_{2}-s_{1}\right| M(M\|B\|)^{n+1} s_{2}^{n} \exp \left(\omega s_{2}\right) / n ! .
\end{aligned}
$$


Finally since $\left\|T\left(s_{2}-\sigma\right)-T\left(s_{1}-\sigma\right)\right\| \leqq 2 M \exp \left(\omega s_{2}\right)$, the integrand on the right converges boundedly to zero for all $\sigma \leqq s_{1}$. Hence $\left\|S_{n+1}\left(s_{2}\right)-S_{n+1}\left(s_{1}\right)\right\| \rightarrow 0$ as $s_{1}, s_{2} \rightarrow s_{0}$ for all $s_{0} \geqq 0$.

Thus we see that being uniformly continuous for $s>0$ is a stable property for a semi-group. However, as we shall show by Example 5.2, being ultimately uniformly continuous is not a stable semi-group property.

Theorem 3.9. Let $\subseteq$ be the open section $\Phi_{1}<\arg z<\Phi_{2}$ where $-\pi / 2 \leqq \Phi_{1}<0$ $<\Phi_{2} \leqq \pi / 2$. Let $T(z)$ be a semi-group of linear operators holomorphic in $\subseteq$ and. such that

$$
\|T[r \exp (i \phi)]\| \leqq K(\phi), \quad 0<r \leqq 1,
$$

where $K(\phi)$ is bounded in every interval $\Phi_{1}+\epsilon<\phi<\Phi_{2}-\epsilon(\epsilon>0)$. Further suppose that $T[r \exp (i \phi)]$ converges strongly to $I$ as $r \rightarrow 0$ for each $\phi \in\left(\Phi_{1}, \Phi_{2}\right)$. Finally let $A$ be the infinitesimal generator of $T(z)$ and let $B \in \mathfrak{E}(\mathfrak{X})$. Then the semi-group of linear operators $S(z)$ generated by $A+B$ on $\mathfrak{D}(A)$ is likewise holomorphic in $\subseteq$, satisfies an inequality of the type (15), and $S[r \exp (i \phi)]$ converges strongly to $I$ as $r \rightarrow 0$ for each $\phi \in\left(\Phi_{1}, \Phi_{2}\right)$.

Let $\Theta$ be the closed convex extension of $\Sigma(A)$, the spectrum of $A$, and let $\sigma(\phi)$ be its function of support. Then $\sigma(\phi)+\delta$ for $\Phi_{1}+\epsilon<\phi<\Phi_{2}-\epsilon$ is the function of support for the closed convex set $\mathfrak{D}(\delta, \epsilon)$. Under the hypothesis of our theorem Hille [2, Theorem 13.5.2] has shown that for each positive $\delta$ and $\epsilon$ there exists an $M(\delta, \epsilon)$ such that $\|R(\lambda ; A)\| \leqq M(\delta, \epsilon) / d(\lambda)$ for each $\lambda$ exterior to $\mathfrak{D}(\delta, \epsilon)$ where $d(\lambda)$ is the distance from $\lambda$ to $\mathfrak{D}$. Thus for $\|B\| M(\delta, \epsilon)$ $<d(\lambda)$ it follows from Lemma 3.1 that $R(\lambda, A+B)$ exists and satisfies the inequality

$$
\| R\left(\lambda ; A+B \| \leqq M(\delta, \epsilon)[d(\lambda)-\|B\| M(\delta, \epsilon)]^{-1} .\right.
$$

If we now adjoin to $\mathfrak{D}(0, \epsilon)$ all points a distance less than or equal to $\|B\| M(\delta, \epsilon)$ from $\mathfrak{D}(0, \epsilon)$, the result is again a convex body $\mathfrak{D}_{\epsilon}^{\prime}$ contained in $\mathfrak{D}(\|B\| M(\delta, \epsilon), \epsilon)$. Further set $d^{\prime}(\lambda)$ equal to the distance from $\lambda$ to $\mathfrak{D}_{\epsilon}^{\prime}$. Then $d^{\prime}(\lambda) \leqq d(\lambda)-\|B\| M(\delta, \epsilon)$. Hence $\|R(\lambda ; A+B)\| \leqq M(\delta, \epsilon) / d^{\prime}(\lambda)$ for all points exterior to $\mathfrak{D}^{\prime}$. Here we suppose $\delta<M(\delta, \epsilon)\|B\|$. If we now employ an argument used by Hille [2, Theorem 13.5.3] we find that $S(z)$ is a semi-group of operators holomorphic in the sector $\Phi_{1}+\epsilon<\phi<\Phi_{2}-\epsilon$ and satisfying an inequality of the type (15). Since $\epsilon>0$ is arbitrary, it follows that $S(z)$ is actually a holomorphic semi-group in all of $\mathfrak{S}$. As Hille shows, $S(s)$ converges strongly to $I$ as $s \rightarrow 0$. From this it follows that for $x \in \mathfrak{D}(A)$

$$
S(z) x=x+\int_{0}^{z} S(\zeta)(A+B) x d \zeta
$$

and hence that $S[r \exp (i \phi)] x \rightarrow x$ for each $\phi \in\left(\Phi_{1}, \Phi_{2}\right)$. Since $S(z)$ also satisfies 
an inequality of the type (15), this remains true for all $x \in \mathfrak{X}$.

We remark that if $T(z)$ is holomorphic in the entire plane, this is equivalent to $T(z)$ having a bounded linear infinitesimal generator $A$. Hence if $B \in \mathbb{E}(\mathfrak{X})$, then $A+B \in \mathbb{E}(\mathfrak{X})$ and therefore $S(z)$ is also holomorphic in the entire plane. Thus being holomorphic in the entire plane is again a stable semi-group property.

4. Completely continuous and weakly completely continuous resolvents. In the semi-groups which occur in the nonsingular problems of mathematical physics, the resolvent of the infinitesimal generator turns out to be completely continuous. It is therefore of interest to study closed linear operators with either completely continuous (c.c.) or weakly completely continuous (w.c.c.) resolvents. We show first of all that both of these are stable properties for the infinitesimal generators of semi-groups of linear bounded operators. In case the resolvent of a closed linear operator is completely continuous, the operator has a pure point spectrum and the eigen-spaces are finite-dimensional. This fact permits us to obtain a simple elementary divisor theory for such operators.

THEOREM 4.1. If $A$ is a closed linear operator on $\mathfrak{X}$ to itself and if for some $\lambda_{0} \in \rho(A), R\left(\lambda_{0} ; A\right)$ is c.c. (or w.c.c.), then $R(\lambda ; A)$ is c.c. (or w.c.c.) for all $\lambda \in \rho(A)$.

This is an immediate consequence of the first resolvent equation (3) since the product of a bounded linear operator and c.c. (or w.c.c.) operator is c.c. (or w.c.c.) and since the sum of two c.c. (or w.c.c.) operators is again c.c. (or w.c.c.).

LeMma 4.2. If $A$ and $B$ are closed linear operators on $\mathfrak{X}$ to itself with nonvacuous resolvent sets, if $\mathfrak{D}(A) \supset \mathfrak{D}(B)$, and if $R(\lambda ; A)$ is c.c. (or w.c.c.), then $R(\mu ; B)$ is also c.c. (or w.c.c.).

Let $\lambda \in \rho(A)$ and $\mu \in \rho(B)$ be fixed. Then $(\lambda I-A)$ is a closed linear operator on $\mathfrak{D}(A)$ and hence $W=(\lambda I-A) R(\mu ; B)$ is closed and linear on $\mathfrak{X}$. By the closed graph theorem due to Banach $[1$, p. 41], $W$ is bounded. Finally $R(\mu ; B)=R(\lambda ; A) W$ is c.c. (or w.c.c.) since it is the product of two linear bounded transformations, one of which is c.c. (or w.c.c.).

THEOREM 4.3. If $A$ is the infinitesimal generator of a semi-group of operators satisfying $(\mathrm{H})$ on $[0, \infty)$ and if $R(\lambda ; A)$ is c.c. (or w.c.c.), then $A+B$ on $\mathfrak{D}(A)$ where $B \in \mathfrak{E}(\mathfrak{X})$ also has a c.c. (or w.c.c.) resolvent.

This is an immediate consequence of the above lemma since both $A$ and $A+B$ are closed linear operators on $\mathfrak{D}(A)$ with nonempty resolvent sets (see Lemma 3.1). The result also follows directly from the representation of $R(\lambda ; A+B)$ given in Lemma 3.1.

Another result similar in character to Lemma 4.2 is given in the following 
theorem which was suggested by a result due to $F$. Rellich on self-adjoint operators $[4$, vol. 118$]$.

THEOREM 4.4. If $U$ and $V$ are linear bounded transformations on $\mathfrak{X}$ to $\mathfrak{Y}$, if the range of $U$ contains that of $V$, and if $U$ is c.c. (or w.c.c.), then $V$ is likewise c.c. (or w.c.c.).

Let $\mathfrak{M} \equiv[x \mid U x=0]$. Then $\mathfrak{M}$ is a closed linear subspace of $\mathfrak{X}$ and defines the quotient space $\mathfrak{X} / \mathfrak{M}$ of $\operatorname{cosets} \tilde{x}$. If we define a norm $\|\tilde{x}\|=\inf [\|x\| \mid x \in \bar{x}]$, then $\mathfrak{X} / \mathfrak{M}$ is again a Banach space. We define $\tilde{U} \tilde{x}=U x$ for $x \in \tilde{x}$. Then $\tilde{U}$ is well defined and linear and possesses an algebraic inverse. Further

$$
\|\tilde{U}\|=\sup [\|\tilde{U} \tilde{x}\| \mid\|\tilde{x}\|<1]=\sup [\|U x\| \mid\|x\|<1]=\|U\|,
$$

so that $\tilde{U}$ is bounded and hence $\widetilde{U}^{-1}$ is closed. Finally $\tilde{U}$ is c.c. (or w.c.c.). For if $\left\|\tilde{x}_{k}\right\| \leqq 1$, then there exist $x_{k} \in \tilde{x}_{k}$ such that $\left\|x_{k}\right\| \leqq 2$ and hence a subsequence of $\tilde{U} \tilde{x}_{k}=U x_{k}$ converges (or converges weakly) in $\mathfrak{Y}$. Now $W=\tilde{U}^{-1} V$ is closed and linear on $\mathfrak{X}$ to $\mathfrak{X} / \mathfrak{M}$ and hence by the closed graph theorem $[1$, p. 41] $W$ is bounded. Finally $V=\widetilde{U} W$ is c.c. (or w.c.c.) since it is the product of two bounded linear operators one of which is c.c. (or w.c.c.).

We remark that Lemma 4.2 is an immediate consequence of Theorem 4.4 if we set $U=R(\lambda ; A)$ and $V=R(\mu ; B)$.

TheOREM 4.5. If $A$ is a closed linear operator on $\mathfrak{X}$ to itself possessing a completely continuous resolvent, then $A$ has a pure point spectrum consisting of isolated points.

By the F. Riesz theory for completely continuous operators (see [1, chap. 10]), it is known that $\left[I+\left(\lambda-\lambda_{0}\right) R\left(\lambda_{0} ; A\right)\right]^{-1}$ exists and belongs to $\mathfrak{E}(\mathfrak{X})$ for all but an at most denumerable set of isolated points $\left\{\lambda_{n}\right\}$. Corresponding to each $\lambda_{n}$ there is one or more nonzero vectors $x_{n}$ such that $\left(\lambda_{n}-\lambda_{0}\right) R\left(\lambda_{0} ; A\right) x_{n}=-x_{n}$. Thus $x_{n} \in \mathfrak{D}(A)$ and $x_{n}=\left(\lambda_{0} I-A\right) R\left(\lambda_{0} ; A\right) x_{n}$ $=\left(\lambda_{0} I-A\right) x_{n} /\left(\lambda_{0}-\lambda_{n}\right)$. In other words, $A x_{n}=\lambda_{n} x_{n}$ so that $\lambda_{n} \in P-\Sigma(A)$. On the other hand if $\lambda \notin\left\{\lambda_{n}\right\}$, then $\left[I+\left(\lambda-\lambda_{0}\right) R\left(\lambda_{0} ; A\right)\right]^{-1}$ exists. $R(\lambda)$ $\equiv R\left(\lambda_{0} ; A\right)\left[I+\left(\lambda-\lambda_{0}\right) R\left(\lambda_{0} ; A\right)\right]^{-1}$ clearly has $\mathfrak{D}(A)$ for its range and is the right inverse of $(\lambda I-A)=\left(\lambda-\lambda_{0}\right) I+\left(\lambda_{0} I-A\right)$. Hence $R(\lambda ; A)=R(\lambda)$ for $\lambda \notin\left\{\lambda_{n}\right\}$. Thus $\Sigma(A)=\left\{\lambda_{n}\right\}$.

We next obtain an elementary divisor theory for $A$, again supposing $R(\lambda ; A)$ to be completely continuous. Let $C_{0}$ be a circle about the point $\lambda_{0}$ $\in \Sigma(A)$ containing no other point of the spectrum. Then

$$
E_{0}=(2 \pi i)^{-1} \int_{C_{0}} R(\lambda ; A) d \lambda
$$

defines a projection operator (see Hille [2, Theorem 5.14.2]). We shall define the range $\mathfrak{M}_{0}$ of $E_{0}$ to be the eigen-space associated with $\lambda_{0}$. 
THEOREM 4.6. Let $A$ be a closed linear operator on $\mathfrak{X}$ to itself with a completely continuous resolvent. For $\lambda_{0} \in \Sigma(A)$ let $\mathfrak{M}_{k}$ be the null space of the operator $\left(\lambda_{0} I-A\right)^{k}$. Then for $k>0, \mathfrak{M}_{k}$ is always of positive finite dimension and there exists an $n>0$ such that $\mathfrak{M}_{k}=\mathfrak{M}_{n}$ for $k \geqq n$ whereas $\mathfrak{M}_{k-1}$ is a proper subset of $\mathfrak{M}_{k}$ for $k \leqq n$.

Since $R(\lambda ; A)$ is holomorphic for $\lambda \in \rho(A)$, the integral in (16) will be the limit in the uniform operator topology of the approximating sums. By hypothesis ${ }^{*} R(\lambda ; A)$ is completely continuous and therefore the same is true of the approximating sums. Therefore $E_{0}$ is the uniform limit of completely continuous operators and is itself completely continuous (see Banach [1, p. 96]). Thus any bounded subset of the range space $\mathfrak{M}_{0}$ must be compact and hence $\mathfrak{M}_{0}$ is finite-dimensional (see Banach $[1, \mathrm{p} .84]$ ). It is easy to show that $A E_{0} x$ exists for all $x$ and that $A E_{0}=E_{0} A$ on $\mathfrak{D}(A)$ (see Hille [2, p. 111]). Thus $\mathfrak{D}(A) \supset \mathfrak{M}_{0}$. We now set $A_{0}=A E_{0}$ and $R_{0}(\lambda)=E_{0} R(\lambda ; A)=R(\lambda ; A) E_{0}$. Then $\left(\lambda E_{0}-A_{0}\right) R_{0}(\lambda)=R_{0}(\lambda)\left(\lambda E_{0}-A_{0}\right)=E_{0}$. Thus relative to $\mathfrak{M}_{0}, R_{0}(\lambda)$ is the resolvent of $A_{0}$. Further it can be shown that $\lambda_{0}$ is the only nonremovable singularity of $R_{0}(\lambda)$ (see Hille [2, Theorem 5.14.2]). Since $\mathfrak{M}_{0}$ is finite-dimensional we have merely to appeal to the algebraic case to see that the theorem is valid for $A_{0}$ on $\mathfrak{M}_{0}$. Suppose for some $x_{0} \in \mathfrak{D}(A)$ that $\left(\lambda_{0} I-A\right) x_{0}=y_{0} \in \mathfrak{M}_{0}$. Then $R(\lambda ; A) y_{0}=R(\lambda ; A) E_{0} y_{0}=E_{0} R(\lambda ; A) y_{0}$ belongs to $\mathfrak{M}_{0}$. Further since $x_{0}=R(\lambda ; A)(\lambda I-A) x_{0}=\left(\lambda-\lambda_{0}\right) R(\lambda ; A) x_{0}+R(\lambda ; A) y_{0}$, we have

$$
x_{0}=(2 \pi i)^{-1} \int_{C_{0}}\left(\lambda-\lambda_{0}\right)^{-1} d \lambda=E_{0} x_{0}+(2 \pi i)^{-1} \int_{C_{0}}\left(\lambda-\lambda_{0}\right)^{-1} R(\lambda ; A) y_{0} d \lambda .
$$

The integral on the right lies in $\mathfrak{M}_{0}$ since this is true of the integrand. Hence $x_{0}$ can be represented as the sum of two elements in $\mathfrak{M}_{0}$ and is itself in $\mathfrak{M}_{0}$. Consequently if $\left(\lambda_{0} I-A\right)^{k} x_{0}=0 \in \mathfrak{M}_{0}$, then $\left(\lambda_{0} I-A\right)^{k-1} x_{0} \in \mathfrak{M}_{0}$ and by induction $x_{0} \in \mathfrak{M}_{0}$. Hence all of the spaces $\mathfrak{M}_{k}($ for $k>0)$ are contained in $\mathfrak{M}_{0}$. Since the theorem was valid for subspaces of $\mathfrak{M}_{0}$, this concludes the proof of the theorem.

Theorem 4.6 is analogous to the results of F. Riesz [5, p. 79] for completely continuous operators $A$. It is also closely connected with the work of A. E. Taylor [6, p. 660] on poles of $R(\lambda ; A)$.

5. Unstable properties. In this section we show by means of examples that the following semi-group properties are not stable under a linear bounded perturbation of the infinitesimal generator: (1) that the semi-group operators $(s>0)$ be generalized nilpotent; (2) that for $s$ sufficiently large the semi-group of operators be uniformly continuous; and (3) that for completely continuous resolvent, the eigen-spaces span $X$.

5.1. If $T(s)$ is a semi-group of linear operators satisfying $(\mathrm{H})$ on $[0, \infty)$ and such that $T(s)$ is generalized nilpotent for $s>0$, then $\omega_{0}=-\infty$ and $R(\lambda ; A)$ is an entire function. If $x_{0} \in \mathfrak{D}(A)$, then it is clear that there exist 
linear bounded transformations $B$ such that $B x_{0}=\lambda_{0} x_{0}-A x_{0}$. For such a $B$, $(A+B) x_{0}=\lambda_{0} x_{0}$ so that $\lambda_{0}$ belongs to the point spectrum of $A+B$. Thus $R(\lambda ; A+B)$ has a singularity at $\lambda_{0}$ and elements of the semi-group generated by $A+B$ are no longer generalized nilpotent for $s>0$.

THEOREM 5.2. The property that a semi-group of linear bounded operators satisfying $(\mathrm{H})$ on $[0, \infty)$ be eventually continuous in the uniform topology is not a stable property.

Suppose the contrary were true and that $A$ is the infinitesimal generator of a semi-group of linear bounded transformations $T(s)$ satisfying $(\mathrm{H})$ on $[0, \infty)$ where $T(s)$ is continuous in the uniform topology for $s$ sufficiently large. Let $B$ be a bounded linear transformation. Then for each complex number $z$ the semi-group $S(z, s)$ generated by $A+z B$ is uniformly continuous for $s \geqq L(z)$. By the usual second category argument there exists a circle $C$ in the $z$-plane and an $L_{0}$ such that $L(z) \leqq L_{0}$ for a set of points $\mathfrak{D}$ dense in $C$. By Corollary 3.6, $S(z, s)$ is bounded if the range of $s$ and $z$ is likewise bounded. Further, by Corollary 3.7, $S(z, s)$ is an entire function of $z$ for each $s \geqq 0$. Hence by the theorem of Vitali as extended by Hille [2, Theorem 3.13.1] it follows that $S(z, s)$ is continuous in the uniform topology when $s \geqq L_{0}$ for each $z$ in the plane. In the present instance, the expansion (12) takes the form

$$
S(z, s)=\sum_{n=0}^{\infty} z^{n} S_{n}(s)
$$

where as before $S_{0}(s)=T(s)$ and $S_{n}(s)=\int_{0}^{s} T(s-\sigma) B S_{n-1}(\sigma) d \sigma$. Thus $S_{n}(s)$ $=(2 \pi i)^{-1} \int_{\Gamma} S(z, s) z^{-(n+1)} d z$ where $\Gamma$ is a circle about the origin. Finally since $\|S(z, s+\Delta)-S(z, s)\|$ converges to zero uniformly for $z \in \Gamma$ as $\Delta \rightarrow 0$ where $s>L_{0}$ (by the Vitali theorem), it follows that $S_{n}(s)$ is likewise continuous in the uniform topology for $s>L_{0}$. Therefore in order to prove the theorem it is sufficient to exhibit a semi-group $T(s)$ satisfying $(H)$ on $[0, \infty)$ and eventually uniformly continuous, along with a bounded linear transformation $B$ such that for any $L_{0}$ there exists an $S_{n}(s)$ which is not continuous in the uniform topology for some $s>L_{0}$.

To this end we set $\mathfrak{X}=\mathfrak{R}(0,1)$ and define $(T(s) f)(\xi)=f(\xi+s)$ for $\xi \leqq 1-s$ and equal to zero otherwise. Then $T(s)$ satisfies $(\mathrm{H})$ on $[0, \infty)$. It is clear that $T(s)=0$ for $s \geqq 1$ so that $T(s)$ is certainly continuous in the uniform topology for $s \geqq 1$. We set $(B f)(\xi)=f(\xi-1 / 2)$ for $\xi \geqq 1 / 2$ and equal to zero otherwise. It is now convenient to introduce the auxiliary function $\phi(\xi)$ defined on $(-\infty, \infty)$ to be zero if $\xi<0$ and if $\xi>1$ and to be one for $0 \leqq \xi \leqq 1$. Then considering $f(\xi) \in \mathfrak{R}(0,1)$ to be extended to the entire line with $f(\xi)=0$ for $\xi \notin[0,1]$, we see that we may also write $(T(s) f)(\xi)=f(\xi+s) \phi(\xi)$ and $(B f)(\xi)$ $=f(\xi-1 / 2) \phi(\xi)$. Thus

$$
T(s-\sigma) B T(\sigma) f=f(\xi+s-1 / 2) \phi(\xi+s-\sigma-1 / 2) \phi(\xi+s-\sigma) \phi(\xi)
$$


and hence

$$
S_{1}(s) f=f(\xi+s-1 / 2) \phi(\xi) \int_{0}^{s} \phi(\xi+s-\sigma-1 / 2) \phi(\xi+s-\sigma) d \sigma .
$$

In general we have

$$
S_{n}(s) f=f(\xi+s-n / 2) K_{n}(\xi, s)
$$

where

$$
K_{n}(\xi, s)=\phi(\xi) \int_{0}^{s} K_{n-1}(\xi+s-\sigma-1 / 2, \sigma) \phi(\xi+s-\sigma) d \sigma .
$$

It is clear from (17) that $S_{n}(s)=0$ for $s \geqq s_{n}=1+n / 2$. We shall now show that $\lim \inf _{s^{\prime} s_{n}^{-}}\left\|S_{n}(s)\right\|>0$. In order to do this we estimate $K_{n}(\xi, s)$. In the first place it is clear that $K_{n}(\xi, s) \geqq 0$. For $1 \leqq s \leqq 3 / 2,0 \leqq \xi \leqq 1 / 2$, and $\xi+s-1 \leqq \sigma$ $\leqq \xi+s-1 / 2$ we see that $0 \leqq \sigma \leqq s$ and that $\phi(\xi+s-\sigma)=1=\phi(\xi+s-\sigma-1 / 2)$. Thus $K_{1}(\xi, s) \geqq 1 / 2$ for $1 \leqq s \leqq 3 / 2$ and $0 \leqq \xi \leqq 1 / 2$. Suppose that $K_{n-1}(\xi, s)$ $\geqq 2^{-n(n-1) / 2}$ for $(n+1) / 2-2^{-(n-1)} \leqq s \leqq(n+1) / 2$ and $0 \leqq \xi \leqq 2^{-(n-1)}$. Then for $n / 2+1-2^{-n} \leqq s \leqq n / 2+1,0 \leqq \xi \leqq 2^{-n}$, and $0 \leqq \xi+s-\sigma-1 / 2 \leqq 2^{-(n-1)}$ we see that the interval $(n+1) / 2-2^{-(n-1)} \leqq \sigma \leqq(n+1) / 2$ has an interval of length at least $2^{-n}$ in common with the above $\sigma$-interval. Further $0 \leqq \xi+s-\sigma \leqq 1$ and hence $\phi(\xi+s-\sigma)=1$. Hence $K_{n}(\xi, s) \geqq 2^{-n} 2^{-n(n-1) / 2}=2^{-n(n+1) / 2}$ for $n / 2$ $+1-2^{-n} \leqq s \leqq n / 2+1$ and $0 \leqq \xi \leqq 2^{-n}$. Finally set $f_{\Delta}(\xi)=1$ for $1-\Delta \leqq \xi \leqq 1$ and equal to zero elsewhere. Then we have by $(17)$ that $S_{n}(n / 2+1-\Delta) f_{\Delta}$ $=f_{\Delta}(\xi+1-\Delta) K_{n}(\xi, n / 2+1-\Delta)$. For $\Delta \leqq 2^{-n},\left\|S_{n}(n / 2+1-\Delta) f_{\Delta}\right\| \geqq 2^{-n(n+1) / 2}$ $\left\|f_{\Delta}\right\|$. Hence $\left\|S_{n}(n / 2+1)-S_{n}(n / 2+1-\Delta)\right\| \geqq 2^{-n(n+1) / 2}$ for $\Delta \leqq 2^{-n}$. Thus $S_{n}(s)$ is not uniformly continuous at $s=n / 2+1$. This concludes the proof of Theorem 5.2.

The regular Sturm-Liouville problem involves a self-adjoint operator on a Hilbert space to itself with completely continuous resolvent. The eigenfunctions span the space and this fact leads to some very useful expansion theorems. Much of the recent work in abstract analysis is an attempt to generalize this familiar situation to the general closed linear transformation on a Banach space to itself. Even if we restrict ourselves to closed linear operators with completely continuous resolvents, the expansion problem need not make sense. Such an operator may have no spectrum and hence no eigenspaces. There are of course many examples where the eigen-spaces do span the space and one might at least hope that this condition has a degree of stability. That this is not the case is illustrated by the example below.

ExAmple 5.3. Let $\mathfrak{X}=\mathfrak{R}_{p}(0,1)$ where $p \geqq 1$. The semi-group of left translations $(T(s) f)(\xi)=f(\xi+s)$ for $\xi \leqq 1-s$ and equal to zero elsewhere satisfies (H) on $[0, \infty)$. Since $T(s)=0$ for $s \geqq 1$, each of the operators $T(s)$ for $s>0$ is generalized nilpotent. The infinitesimal generator $A$ is the derivative operator acting on the domain $\mathfrak{D}(A)$ of all absolutely continuous functions on $[0,1]$ 
with derivatives in $\mathfrak{X}$ and which vanish at $\xi=1$. The resolvent $R(\lambda ; A) f$ $=\int_{\xi}^{1} \exp [\lambda(\xi-\sigma)] f(\sigma) d \sigma$ is clearly completely continuous and entire in $\lambda$. Thus $A$ has no eigen-spaces whatever. To the operator $A$ we add the onedimensional linear bounded operator $(B f)(\xi)=4 \int_{0}^{1} f(\sigma) d \sigma$. The operator $C=A$ $+B$ is closed and linear on $\mathfrak{D}(C) \equiv \mathfrak{D}(A)$ and generates a semi-group of linear operators. The resolvent of $C$ is of course still completely continuous; however $R(\lambda, C)$ has an infinite set of singular points. These are the solutions of the indicial equation $(\lambda-2)^{2}=4 \exp (-\lambda)$ of the differential-integral equation $\lambda y-y^{\prime}-4 \int_{0}^{1} y(\sigma) d \sigma=0$ with boundary condition $y(1)=0$.

We shall now show that the eigen-spaces of $C$ span $\mathfrak{X}$. Since $\mathfrak{D}\left(C^{k}\right)$ is dense in $\mathfrak{X}$ for each $k>0$ (see Hille [2, Theorem 11.5.1]) it will be sufficient to show that any $f \in \mathfrak{D}\left(C^{4}\right)$ can be approximated by the elements of the eigenspaces. In particular it will be sufficient to show that for $f \in \mathfrak{D}\left(C^{4}\right)$

$$
F_{n} f=(2 \pi i)^{-1} \int_{\Gamma_{n}} R(\lambda ; C) f d \lambda \rightarrow f
$$

as $n \rightarrow \infty$ for a set of expanding contours $\Gamma_{n}$. Now by repeated application of (4) we obtain for $f \in \mathfrak{D}\left(C^{4}\right)$

$$
R(\lambda ; C) f=f \lambda^{-1}+C f \lambda^{-2}+C^{2} f \lambda^{-3}+C^{3} f \lambda^{-4}+R(\lambda ; C) C^{4} f \lambda^{-4} .
$$

Hence if $\Gamma_{n}$ encircles the origin, we obtain

$$
(2 \pi i)^{-1} \int_{\mathrm{r}_{n}} R(\lambda ; C) f d \lambda=f+(2 \pi i)^{-1} \int_{\mathrm{r}_{n}} R(\lambda ; C) C^{4} f \lambda^{-4} d \lambda .
$$

In order to establish (18) it is therefore sufficient to show that

$$
\left\|\int_{\mathrm{r}_{n}} R(\lambda ; C) g \lambda-4 d \lambda\right\| \rightarrow 0
$$

as $n \rightarrow \infty$ for each $g \in \mathfrak{X}$.

A straightforward calculation yields

$$
\begin{aligned}
p(\lambda) R(\lambda ; C) g & =(\lambda-2)^{2} \int_{\xi}^{1} \exp [-\lambda(\sigma-\xi)] g(\sigma) d \sigma \\
& +4\{1-\exp [-\lambda(1-\xi)]\} \int_{0}^{1} g(\sigma) d \sigma \\
& +4 \exp (-\lambda) \int_{0}^{\xi} \exp [\lambda(\xi-\sigma)] g(\sigma) d \sigma \\
& -4 \int_{0}^{1} \exp (-\lambda \sigma) g(\sigma) d \sigma
\end{aligned}
$$

where $p(\lambda)=(\lambda-2)^{2}-4 \exp (-\lambda)$. For $\Gamma_{n}$ we shall choose circles of radius 
$r_{n}$ about $\lambda=2$ with $r_{n} \rightarrow \infty$ as $n \rightarrow \infty$. We shall require an estimate of $p(\lambda)$ for $\lambda \in \Gamma_{n}$. Since the absolute value of $p(\lambda)$ remains unchanged if we substitute $\lambda$ conjugate for $\lambda$, we need only consider $\lambda$ in the upper half-plane. Let $\lambda-2=r \exp (i \theta)=x+i y$. Then the zeros of $p(\lambda)$ in the upper half plane occur where (a) $r^{2}=4 \exp (-x-2)$ and (b) $2 \theta=-y+2 n \pi$ for some positive or negative integer $n$. Consider first $\left[x=-2-\log \left(r^{2} / 4\right), y=\left(r^{2}-x^{2}\right)^{1 / 2}\right]$ which is the solution of (a) as a function of $r$. As $r$ increases, $x$ decreases, $y$ increases, and $\theta$ decreases toward $\pi / 2$. Hence for large increasing $r, \theta$ is practically stationary so that (b) will be satisfied by increasing values of $y$ approximately $2 \pi$ apart. We now choose for the $r_{n}$ 's successive values of $r$ for which $y$ satisfies $y \equiv-2 \theta+\pi$ (modulo $2 \pi$ ) when $x$ satisfies (a). We shall show for $|\lambda-2|=r_{n}$ that

$$
|p(\lambda)| \geqq(1 / 4)\left[r^{2}+4|\exp (-\lambda)|\right]=(1 / 4)\left[r^{2}+4 \exp (-x-2)\right] \text {. }
$$

In general $|p(\lambda)|>\left|r^{2}-4 \exp (-x-2)\right|$ and hence (21) is satisfied whenever $r^{2} \geqq 8 \exp (-x-2)$ or whenever exp $(-x-2) \geqq r^{2} / 2$. Thus (21) is satisfied for $x$ outside the interval $-2-\log \left(r^{2} / 2\right) \leqq x \leqq-2-\log \left(r^{2} / 8\right)$. The width of this $x$-interval is just $\log 4$ and it contains the value $x=-2-\log \left(r^{2} / 4\right)$. For fixed $r$, the corresponding change in $y$ is roughly $\log \left(r^{2} / 4\right) \log (4) r^{-1}$ which goes to zero as $r \rightarrow \infty$. Likewise the corresponding change in $\theta$ is less than $\log 4 / r$ which also goes to zero as $r \rightarrow \infty$. Hence for $r=r_{n}$ and large $n$, the vectors $(\lambda-2)^{2}$ and $4 \exp (-\lambda)$ are roughly $\pi$ radians out of phase in the doubtful interval. Thus for large $n$, the angle between these vectors will certainly remain greater than $\pi / 2$, so that even in this doubtful interval the inequality (21) remains valid for $n$ sufficiently large. It is now clear from (20) and (21) that each term in $R(\lambda, C) g$ is in norm $O\left(r_{n}^{2}\right)$ as $r_{n} \rightarrow \infty$. Hence the integral of $\lambda^{-4} R(\lambda ; C) g$ about $\Gamma_{n}$ goes to zero as $n \rightarrow \infty$. This concludes the proof of the fact that the eigen-spaces of $C$ span $\mathfrak{X}$.

6. A differential equation. A semi-group of linear operators $T(s)$, satisfying (H) on $[0, \infty)$ and with infinitesimal generator $A$, solves the differential equation $d T(s) x / d s=A T(s) x$ for $x \in \mathfrak{D}(A)$ and with initial condition $T(0)=1$. An instance of this differential equation would be a linear partial differential equation with $s$-invariant coefficients. The fact that the coefficients must be $s$-invariant constitutes a serious limitation on the applicability of the theory and it is clearly desirable that $A$ be permitted to vary with $s$. We are therefore led to the following problem: Given a one-parameter family of closed linear operators $A(s)$ with domains dense in $\mathfrak{X}$, to find a one-parameter family of linear bounded operators $U(s)$ strongly continuous for $s \geqq 0$ such that $U(0)=I$ and $d U(s) x / d s=A(s) U(s) x$ for all $x$ in a given dense domain. In this section we shall present a solution to a rather restricted instance of this general problem.

LEMMA 6.1. Let $T(s)$ be a semi-group of linear operators satisfying $(\mathrm{H})$ on $[0, \infty)$. For $f(s)$ strongly continuous on $[0, \infty)$ to $\mathfrak{X}, g(s)=\int_{0}^{s} T(s-\sigma) f(\sigma) d \sigma$ $=\int_{0}^{s} T(\sigma) f(s-\sigma) d \sigma$ exists and is itself strongly continuous on $[0, \infty)$ to $\mathfrak{X}$. If 
$f(s)$ is strongly continuously differentiable, then so is $g(s)$ and

$$
\begin{aligned}
d g(s) / d s & =T(s) f(0)+\int_{0}^{s} T(s-\sigma) f^{\prime}(\sigma) d \sigma \\
& =f(s)+A \int_{0}^{s} T(s-\sigma) f(\sigma) d \sigma .
\end{aligned}
$$

Since $\|T(s)\| \leqq M \exp (\omega s)$, it is clear that $T(s-\sigma) f(\sigma)$ is strongly continuous in $\sigma \in[0, s]$ whenever the same is true of $f(\sigma)$. In this case $g(s)$ $=\int_{0}^{s} T(s-\sigma) f(\sigma) d \sigma$ will exist in the strong topology and be equal to $\int_{0}^{s} T(\sigma) f(s-\sigma) d \sigma$. Further( $\left(^{(3)}\right.$

$$
\begin{aligned}
g(s+\Delta)-g(s)= & \int_{0}^{8} T(\sigma)[f(s+\Delta-\sigma)-f(s-\sigma)] d \sigma \\
& +\int_{s}^{s+\Delta} T(\sigma) f(s+\Delta-\sigma) d \sigma .
\end{aligned}
$$

The integrand in the first term converges pointwise to zero and remains bounded in the norm as $\Delta \rightarrow 0$. The integrand in the second term also remains bounded in norm as $\Delta \rightarrow 0$. Hence $\|g(s+\Delta)-g(s)\| \rightarrow 0$ as $\Delta \rightarrow 0$. If we further assume that $f(s)$ is strongly differentiable with bounded derivative, then, dividing (23) termwise by $\Delta$, we first note that $\| T(\sigma)\left[\{f(s+\Delta-\sigma)-f(s-\sigma)\} \Delta^{-1}\right.$ $\left.-f^{\prime}(s-\sigma)\right] \|$ converges boundedly to zero for $\sigma<s$, and second we note for $\sigma \in[s, s+\Delta]$ that $\|T(\sigma) f(s+\Delta-\sigma)-T(s) f(0)\| \rightarrow 0$ as $\Delta \rightarrow 0$. The first equality in (22) now follows. We next write the incremental ratio as

$$
\begin{aligned}
{[g(s+\Delta)-g(s)] \Delta^{-1}=} & {[T(\Delta)-I] \Delta^{-1} \int_{0}^{s} T(s-\sigma) f(\sigma) d \sigma } \\
& +\Delta^{-1} \int_{s}^{s+\Delta} T(s+\Delta-\sigma) f(\sigma) d \sigma .
\end{aligned}
$$

As above, $\Delta^{-1} \int_{s}^{s+\Delta} T(s+\Delta-\sigma) f(\sigma) d \sigma \rightarrow f(s)$. Further we already know that $d g(s) / d s$ exists. Hence $\int_{0}^{s} T(s-\sigma) f(\sigma) d \sigma \in \mathfrak{D}(A)$ so that the second equality of (22) is likewise valid. The strong continuity of $d g(s) / d s$ follows from the first part of the theorem using the first representation of $d g(s) / d s$ given by (22).

TheOREM 6.2. Let $A$ be the infinitesimal generator of a semi-group of linear operators $T(s)$ satisfying $(\mathrm{H})$ on $[0, \infty)$. Let $B(s)$ be a strongly continuously differentiable function on $[0, \infty)$ to $\mathfrak{E}(\mathfrak{X})$. Then there is a unique one-parameter family of bounded linear operators $U(s)$ strongly continuous on $[0, \infty)$ such that $U(0)=I$ and for $x \in \mathfrak{D}(A), U(s) x$ is strongly continuously differentiable and $\Delta<0$.

(3) For convenience we can define $f(s)=f(0)$ for $s<0$. This makes (23) meaningful for 


$$
d U(s) x / d s=[A+B(s)] U(s) x .
$$

This solution has the representation

$$
U(s)=\sum_{n=0}^{\infty} S_{n}(s)
$$

where $S_{0}(s)=T(s)$ and $S_{n}(s)=\int_{0}^{s} T(s-\sigma) B(\sigma) S_{n-1}(\sigma) d \sigma$.

We employ the method of successive approximations, defining

$$
U_{0}(s)=T(s), U_{n}(s) x=T(s) x+\int_{0}^{s} T(s-\sigma) B(\sigma) U_{n-1}(\sigma) x d \sigma
$$

and

$$
\begin{aligned}
W_{0}(s) & =U_{0}(s), \\
W_{n}(s) x & =U_{n}(s) x-U_{n-1}(s) x=\int_{0}^{s} T(s-\sigma) B(\sigma) W_{n-1}(\sigma) x d \sigma .
\end{aligned}
$$

In the first place $B(s) U_{0}(s) x$ is clearly strongly continuous for all $x \in \mathfrak{X}$. Hence by Lemma $6.1, U_{1}(s) x$ is well defined and strongly continuous. By an inductive argument we see that $U_{n}(s) x$ is likewise strongly continuous. Now $\left\|W_{0}(s)\right\| \leqq M \exp (\omega s)$. Let $K_{s}=\sup \left[\|B(\sigma)\|,\left\|B^{\prime}(\sigma)\right\| \mid \sigma \in[0, s]\right]$. Then it is readily seen that

$$
\left\|W_{n}(s)\right\| \leqq M^{n+1} K_{s}^{n} \exp (\omega s) s^{n} / n !
$$

Hence $U_{n}(s)=\sum_{k=0}^{n} W_{k}(s)$ converges uniformly in every finite interval (in the uniform topology) to a strongly continuous function $U(s)$ on $[0, \infty)$ to $\mathfrak{E}(\mathfrak{X})$. Since $U_{n}(0)=I$, it follows that $U(0)=I$. Finally taking the limit on both sides of (26) we obtain

$$
U(s) x=T(s) x+\int_{0}^{s} T(s-\sigma) B(\sigma) U(\sigma) x d \sigma .
$$

Up to this point we have used only the fact that $B(s)$ is strongly continuous. We show in Example 6.4 that this is not sufficient in order that $U(s)$ be a solution for (24). On the other hand, if it were known for $x \in \mathfrak{D}(A)$ that $U(s) x$ is strongly continuously differentiable, then we would have

$$
\begin{aligned}
{[B(s+\Delta) U(s+\Delta) x-B(s)} & U(s) x] \Delta^{-1} \\
= & B(s+\Delta)\left\{[U(s+\Delta) x-U(s) x] \Delta^{-1}\right\} \\
& +[B(s+\Delta)-B(s)] \Delta^{-1} U(s) x \\
\rightarrow & B(s) U^{\prime}(s) x+B^{\prime}(s) U(s) x .
\end{aligned}
$$

Hence $B(s) U(s) x$ would likewise be strongly continuously differentiable (here we use the fact that $B(s)$ is strongly continuously differentiable) and 
by Lemma 6.1 we could differentiate both sides of (29) to obtain

$$
\begin{aligned}
U^{\prime}(s) x & =A T(s) x+A \int_{0}^{s} T(s-\sigma) B(\sigma) U(\sigma) x d \sigma+B(s) U(s) x \\
& =[A+B(s)] U(s) x .
\end{aligned}
$$

It is clear from this that $U(s)$ would then take $\mathfrak{D}(A)$ into itself. Thus aside from the uniqueness proof, it remains only to show that $U(s) x$ is strongly continuously differentiable for $x \in \mathfrak{D}(A)$.

It is clear that $W_{0}(s) x$ is strongly continuously differentiable for $x \in \mathfrak{D}(A)$. Assuming that this is true of $W_{n}(s) x$, then as in (30) it is easily shown to be true of $B(s) W_{n}(s) x$ and hence by Lemma 6.1 it will be true of $W_{n+1}(s) x$. Again by Lemma 6.1 we have

$$
\begin{aligned}
W_{n}^{\prime}(s) x= & T(s) B(0) W_{n-1}(0) x+\int_{0}^{s} T(s-\sigma) B^{\prime}(\sigma) W_{n-1}(\sigma) x d \sigma \\
& +\int_{0}^{s} T(s-\sigma) B(\sigma) W_{n-1}^{\prime}(\sigma) x d \sigma .
\end{aligned}
$$

Thus

$$
\left\|W_{0}^{\prime}(s) x\right\| \leqq M \exp (\omega s)\|A x\|
$$

and

$$
\begin{aligned}
\left\|W_{1}^{\prime}(s) x\right\| \leqq & M K_{8} \exp (\omega s)\|x\| \\
& +M^{2} K_{8} s \exp (\omega s)\|x\|+M^{2} K_{8} s \exp (\omega s)\|A x\| \\
\leqq & M^{2} K_{8} \exp (\omega s)(1+s) C
\end{aligned}
$$

where $C=\|x\|+\|A x\|$. Making use of (28) we obtain by induction that

$$
\left\|W_{n}^{\prime}(s) x\right\| \leqq M^{n+1} K_{s}^{n} \exp (\omega s)\left[\left(s^{n-1}+s^{n}\right) /(n-1) !\right] C .
$$

Thus $\sum_{n=0}^{\infty} W_{n}^{\prime}(s) x$ converges uniformly in every finite interval to a strongly continuous function which, by the usual argument, is $U^{\prime}(x)$.

In the uniqueness proof we shall need only the strong continuity of $B(s)$. Since $\mathfrak{D}(A)$ is dense in $\mathfrak{X}$, it will be sufficient to show that if for $x \in \mathfrak{D}(A)$, $U(s) x$ solves the differential equation (24) with initial condition $U(0)=0$, then $U(s) x=0$ for all $s \geqq 0$. In other words it is sufficient to consider a strongly continuously differentiable function $y(s)$ on $[0, \infty)$ to $\mathfrak{D}(A)$ such that $y(0)=0$ and $d y(s) / d s=[A+B(s)] y(s)$. Operating on both sides of this equation by $T(s-\sigma)$ and integrating gives

$$
\int_{0}^{s} T(s-\sigma) y^{\prime}(\sigma) d \sigma=\int_{0}^{8} T(s-\sigma) A y(\sigma) d \sigma+\int_{0}^{s} T(s-\sigma) B(\sigma) y(\sigma) d \sigma .
$$


As in (30) it is easy to show for $\sigma \in[0, s]$ that

$$
[T(s-\sigma) y(\sigma)]^{\prime}=-T(s-\sigma) A y(\sigma)+T(s-\sigma) y^{\prime}(\sigma) .
$$

We therefore obtain from (31)

$$
y(s)=\int_{0}^{s} T(s-\sigma) B(\sigma) y(\sigma) d \sigma .
$$

Let $m_{s}=\sup [\|y(\sigma)\| \mid \sigma \in[0, s]]$. Then $m_{s} \leqq M K_{s} m_{s} \int_{0}^{s} \exp (\omega \sigma) d \sigma$ and for $s$ such that $M K_{s} \int_{0}^{s} \exp (\omega \sigma) d \sigma<1$ it is clear that $m_{s}=0$. Since there is nothing special about the origin in this argument, this clearly implies that $y(s)=0$ for all $s \geqq 0$.

It is worth noting that if a solution $V(s)$ to (24) exists for $B(s)$ merely strongly continuous, then the method of successive approximations which we employed will lead to this solution. For operating on both sides of (24) by $T(s-\sigma)$ and integrating gives for $x \in \mathfrak{D}(A)$

$$
V(s) x=T(s) x+\int_{0}^{s} T(s-\sigma) B(\sigma) V(\sigma) x d \sigma .
$$

On the other hand the method of successive approximations yields $U(s)$ which satisfies (29). The difference $y(s)=V(s) x-U(s) x$ satisfies (32) and vanishes at $s=0$. Thus the uniqueness argument shows that this difference is identically zero.

THEOREM 6.3. Let $A$ be the infinitesimal generator of a semi-group of linear operators $T(s)$ satisfying $(H)$ on $[0, \infty)$. Let $B(s)$ and $f(s)$ be strongly continuously differentiable functions on $[0, \infty)$ to $\mathfrak{E}(\mathfrak{X})$ and $\mathfrak{X}$ respectively. Then for each $x \in \mathfrak{D}(A)$ there exists a unique continuously differentiable function $y(s)$ on $[0, \infty)$ to $\mathfrak{X}$ such that

$$
y^{\prime}(s)=[A+B(s)] y(s)+f(s)
$$

and $y(0)=x$. This solution has the form

$$
y(s)=U(s) x+\sum_{n=0}^{\infty} w_{n}(s)
$$

where $U(s)$ is given by $(25), w_{0}(s)=\int_{0}^{s} T(s-\sigma) f(\sigma) d \sigma$, and

$$
w_{n}(s)=\int_{0}^{s} T(s-\sigma) B(\sigma) w_{n-1}(\sigma) d \sigma .
$$

It follows from Lemma 6.1 that $w_{0}(s)$ is strongly continuously differentiable and hence by induction that $w_{n}(s)$ is likewise. In fact

$$
w_{0}^{\prime}(s)=T(s) f(0)+\int_{0}^{s} T(s-\sigma) f^{\prime}(\sigma) d \sigma
$$




$$
w_{n}^{\prime}(s)=\int_{0}^{s} T(s-\sigma) B^{\prime}(\sigma) w_{n-1}(\sigma) d \sigma+\int_{0}^{s} T(s-\sigma) B(\sigma) w_{n-1}^{\prime}(\sigma) d \sigma .
$$

It is now easy to obtain the estimates

$$
\begin{aligned}
& \left\|w_{n}(s)\right\| \leqq M^{n+1} K_{s}^{n} \exp (\omega s) C_{s} s^{n+1} /(n+1) !, \\
& \left\|w_{n}^{\prime}(s)\right\| \leqq M^{n+1} K_{s}^{n} \exp (\omega s) C_{s}\left(s^{n}+s^{n+1}\right) / n !
\end{aligned}
$$

where $C_{s}=\sup \left[\|f(\sigma)\|,\left\|f^{\prime}(\sigma)\right\| \mid \sigma \in[0, s]\right]$. If we set $w(s)=\sum_{n=0}^{\infty} w_{n}(s)$, then as in the previous theorem $w(s)$ is strongly continuously differentiable and $w^{\prime}(s)=\sum_{n=0}^{\infty} w_{n}^{\prime}(s)$. Further $w(0)=0$. From the definition of $w_{n}(s)$ and the uniform convergence of the series $\sum_{n=0}^{\infty} w_{n}(s)$ in every finite interval, it follows that

$$
w(s)=w_{0}(s)+\int_{0}^{s} T(s-\sigma) B(\sigma) w(\sigma) d \sigma .
$$

Since $w(s)$ is strongly continuously differentiable we may, by Lemma 6.1, differentiate (37) termwise. This shows immediately that $w(s)$ is a particular solution of (34). Thus by Theorem 6.2, $U(s) x+w(s)$ is a solution for our problem. The uniqueness follows precisely as in the uniqueness proof of Theorem 6.2.

The particular solution $w(s)$ can also be put in a more suggestive form. Let $U(s, \tau)$ be the solution of (24) for $s \geqq \tau$ with initial condition $U(\tau, \tau)=I$. Then by Theorem 6.2, $U(s, \tau)=\sum_{n=0}^{\infty} S_{n}(s, \tau)$ where $S_{0}(s, \tau)=T(s-\tau)$ and $S_{n}(s, \tau)=\int_{\tau}^{s} T(s-\sigma) B(\sigma) S_{n-1}(\sigma, \tau) d \sigma$. On the other hand $w_{0}(s)=\int_{0}^{s} S_{0}(s, \tau) f(\tau) d \tau$. Further

$$
\begin{aligned}
\int_{0}^{s} S_{n}(s, \tau) f(\tau) d \tau & =\int_{0}^{s} \int_{\tau}^{s} T(s-\sigma) B(\sigma) S_{n-1}(\sigma, \tau) f(\tau) d \sigma d \tau \\
& =\int_{0}^{s} T(s-\sigma) B(\sigma) \int_{0}^{\sigma} S_{n-1}(\sigma, \tau) f(\tau) d \tau d \sigma .
\end{aligned}
$$

Hence by induction $w_{n}(s)=\int_{0}^{s} S_{n}(s, \tau) f(\tau) d \tau$. Finally because of the uniform convergence (in the strong topology) of all series involved we have

$$
w(s)=\int_{0}^{s} U(s, \tau) f(\tau) d \tau .
$$

EXAMPLE 6.4. We now show by example that strong continuity of $B(s)$ in the hypothesis to Theorem 6.2 is not sufficient. Let $\mathfrak{X}=\mathfrak{R}_{p}(0,1)$ with $p \geqq 1$ and set $(T(s) f)(\xi)=f(\xi+s)$ for $\xi \leqq 1-s$ and equal to zero elsewhere. If $0 \leqq s$ $\leqq 1 / 2$, we set $(B(s) f)(\xi)=f(\xi)$ for $1 / 2-s \leqq \xi \leqq 1$ and equal to zero elsewhere; whereas if $s>1 / 2$, we set $B(s)=I$. It is clear that $B(s)$ is strongly continuous on $[0, \infty)$. If $(24)$ has a solution $U(s)$, then as we noted at the end of the 
proof of Theorem 6.2, this solution will have the representation (25). Suppose now that $f \in \mathfrak{D}(A)$; here $\mathfrak{D}(A)$ consists of all absolutely continuous functions which vanish at $\xi=1$ and whose derivatives again belong to $\mathfrak{X}$. Then it is easy to verify for $0 \leqq s \leqq 1 / 2$ and $n>0$ that $S_{n}(s) f=s^{n} f(\xi+s) / n$ ! for $1 / 2-s \leqq \xi<1-s$ and vanishes elsewhere. Hence $U(s) f=f(\xi+s)$ for $0 \leqq \xi<1 / 2$ $-s ;=\exp (s) f(\xi+s)$ for $1 / 2-s \leqq \xi \leqq 1-s$; and $=0$ for $1-s<\xi \leqq 1$. In general for $f \in \mathfrak{D}(A), U(s) f$ will have a jump discontinuity at $(1 / 2-s)$ and hence will not itself belong to $\mathfrak{D}(A)$. On the other hand (24) has no meaning if $U(s) f$ does not belong to $\mathfrak{D}(A)$.

\section{REFERENCES}

1. S. Banach, Theorie des operations lineaires, Warsaw, 1932.

2. Einar Hille, Functional analysis and semi-groups, Amer. Math. Soc. Colloquium Publications, vol. 31, New York, 1948.

3. J. D. Newburgh, The variation of spectra, Duke Math. J. vol. 18 (1951) pp. 165-175.

4. F. Rellich, Störungstheorie der Spektralzerlegung, Math. Ann. vol. 113 (1936) pp. 600619 and pp. 677-685; vol. 116 (1939) pp. 555-570; vol. 117 (1940) pp. 356-382; and vol. 118 (1942) pp. 462-484.

5. F. Riesz, Über lineare Funktionalgleichungen, Acta Math. vol. 41 (1918) pp. 71-98.

6. A. E. Taylor, Analysis in a complex Banach space, Bull. Amer. Math. Soc. vol. 49 (1943) pp. $652-669$.

7. František Wolf, Analytic perturbation of operators in Banach spaces, Math. Ann. vol. 124 (1952) pp. 317-333.

8. Kosaku Yosida, On the differentiability and the representation of the one-parameter semigroup of linear operators, Journal of the Mathematical Society of Japan vol. 1 (1948) pp. 15-21.

University of Southern California, Los ANgeles, Calif. 